\title{
Hyperhomocysteinemia: a risk factor in unexplained infertility
}

\section{Preeti Dubey, Neena Gupta, Seema Dwivedi, Neelam Swaroop, Pavika Lal, Vandana Thawani*}

Department of Obstetrics \& Gynecology, G.S.V.M. Medical College, Kanpur-208002, U.P., India

Received: 16 February 2013

Accepted: 22 March 2013

\section{*Correspondence:}

Dr. Vandana Thawani,

E-mail: vandana.thawani@yahoo.com,vandana.thawani@gmail.com

(C) 2013 Dubey $\mathrm{P}$ et al. This is an open-access article distributed under the terms of the Creative Commons Attribution License, which permits unrestricted use, distribution, and reproduction in any medium, provided the original work is properly cited.

\begin{abstract}
Background: To study the role of hyperhomocysteinemia in unexplained infertility and the impact of its correction with vitamin and mineral supplementation.

Methods: Total of 60 patients were included with 30 patients in case and 30 patients in control groups. Cases included patients with history of inability to conceive with frequent regular unprotected intercourse for at least 1 year. Controls included age matched parous females with at least one live birth and no history of abortions. Among the patients of unexplained infertility with hyperhomocysteinemia, homocysteine lowering agents were given and outcome studied in the form of lowering of homocysteine levels and number of conceptions.

Results: The mean age was 28.1 years in study and 29.5 years in the control group .Mean level of serum homocysteine was significantly higher in study group than normal fertile women i.e. $20.5 \mu \mathrm{mol} / 1$ and $10.9 \mu \mathrm{mol} / 1$ respectively. Among the patients of unexplained infertility, 22(73.3\%) were found to have range above the normal healthy levels. In these patients homocysteine lowering agents were given for 6 weeks and lowering of mean homocysteine levels was observed which was $10.4 \mu \mathrm{mol} / \mathrm{l}$. Six (27.3\%) patients conceived spontaneously during the follow up period.

Conclusions: Serum homocysteine levels are inversely correlated with infertility. Homocysteine lowering agents have a favourable impact on the outcome of infertility and their use is suggested in cases of unexplained infertility associated with hyperhomocysteinemia.
\end{abstract}

Keywords: Hyperhomocysteinemia, Unexplained infertility, Homocysteine lowering agents, Female infertility.

\section{INTRODUCTION}

Infertility implies an apparent failure of a couple to conceive after one year of unprotected regular intercourse. This is based on the observation that $90 \%$ of the normal couples achieve conception within a year. ${ }^{1}$ Infertility is primary if conception has never occurred and secondary if the patient fails to conceive after having achieved a previous conception. The incidence of infertility in any community varies between $10 \%$ and $15 \% .^{2}$

Unexplained infertility is a term applied to an infertile couple whose standard investigations are normal.
Therefore, the diagnosis of unexplained infertility is one of exclusion. ${ }^{3}$ The incidence of unexplained infertility is approximately $15-17 \%$ among the infertile couples. ${ }^{4}$ The cause of infertility is attributed to female factors in 40$55 \%$, male factors in $20-30 \%$, both male and female in $10-40 \%$ and unexplained in $10-20 \%$. $^{5}$

Hyperhomocysteinemia (HHCY) has been underlined as an emerging risk factor for several diseases such as arterial and/or venous thrombosis, adverse pregnancy outcome, congenital malformations and vascular dementia but its role in unexplained infertility is yet under evaluation. ${ }^{6-11}$ 


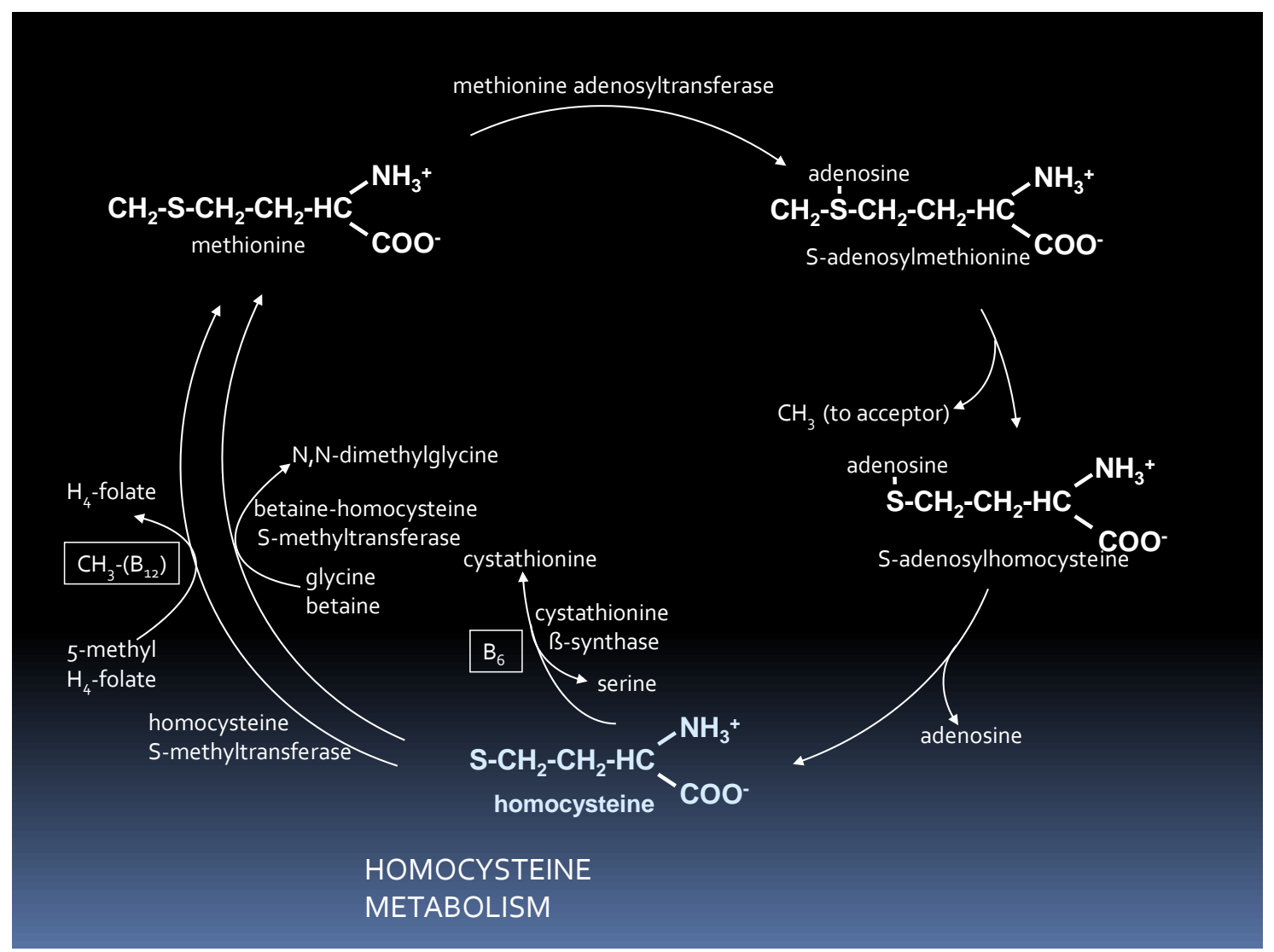

Figure 1: Homocysteine metabolic pathway.

Homocysteine is metabolized through two pathways: remethylation and transsulfuration. ${ }^{12}$ Remethylation requires folate and B12 coenzymes; transsulfuration requires pyridoxal-5'-phosphate, the B6 coenzyme. The remethylation pathway requires vitamin B12, folate, and the enzyme 5, 10-methylenetetrahydrofolate reductase (MTHFR). In kidney and liver, homocysteine is also remethylated by the enzyme betaine homocysteine methyltransferase (BHMT), which transfers a methyl group to homocysteine via the demethylation of betaine to dimethylglycine (DMG). The transulfuration pathway requires the enzyme cystathionine-synthase (CBS) and vitamin B6 (pyridoxal-5'- phosphate). Once formed from cystathionine, cysteine can be utilized in protein synthesis and glutathione (GSH) production. Active folate, known as 5-MTHF or 5-methyltetrahydrofolate, works in concert with vitamin B12 as a methyl-group donor in the conversion of homocysteine back to methionine. Normally, about $50 \%$ of homocysteine is remethylated; the remaining homocysteine is transsulfurated to cysteine, which requires vitamin B6 as a co-factor. This pathway yields cysteine, which is then used by the body to make glutathione, a powerful antioxidant that protects cellular components against oxidative damage.

Vitamin B2 (riboflavin) and magnesium are also involved in homocysteine metabolism. Thus a person needs different type of vitamins B to keep homocysteine levels low and allow for it to be properly transformed into helpful antioxidants like glutathione. Without adequate levels of vitamin B2, B6, B12, folate and magnesium, dangerous levels of homocysteine may build up in the body. Elevations in intracellular homocysteine concentrations with corresponding increase in blood levels can result from augmented production or reduced metabolism. Elevation in serum levels of homocysteine are typically caused either by genetic defects in the enzymes involved in metabolism or by nutritional deficiencies in vitamin cofactors. It has been observed in literature that these nutritional deficiencies contribute to approximately two thirds of all cases of hyperhomocysteinemia.

Homocysteine has been shown to induce vascular inflammation by enhancing the expression of proinflammatory cytokines, such as monocyte chemoattractant protein 1 (MCP-1), which regulates migration and activation of monocytes/macrophages, and interleukin 8 (IL-8), which is an important chemoattractant for neutrophils and T-lymphocyte. ${ }^{13}$ Second, homocysteine decreases the bioavailability of nitric oxide (NO), which is one of the major endotheliumdependent vasodilators that is produced by the endothelial isoform of nitric oxide synthase (eNOS). 


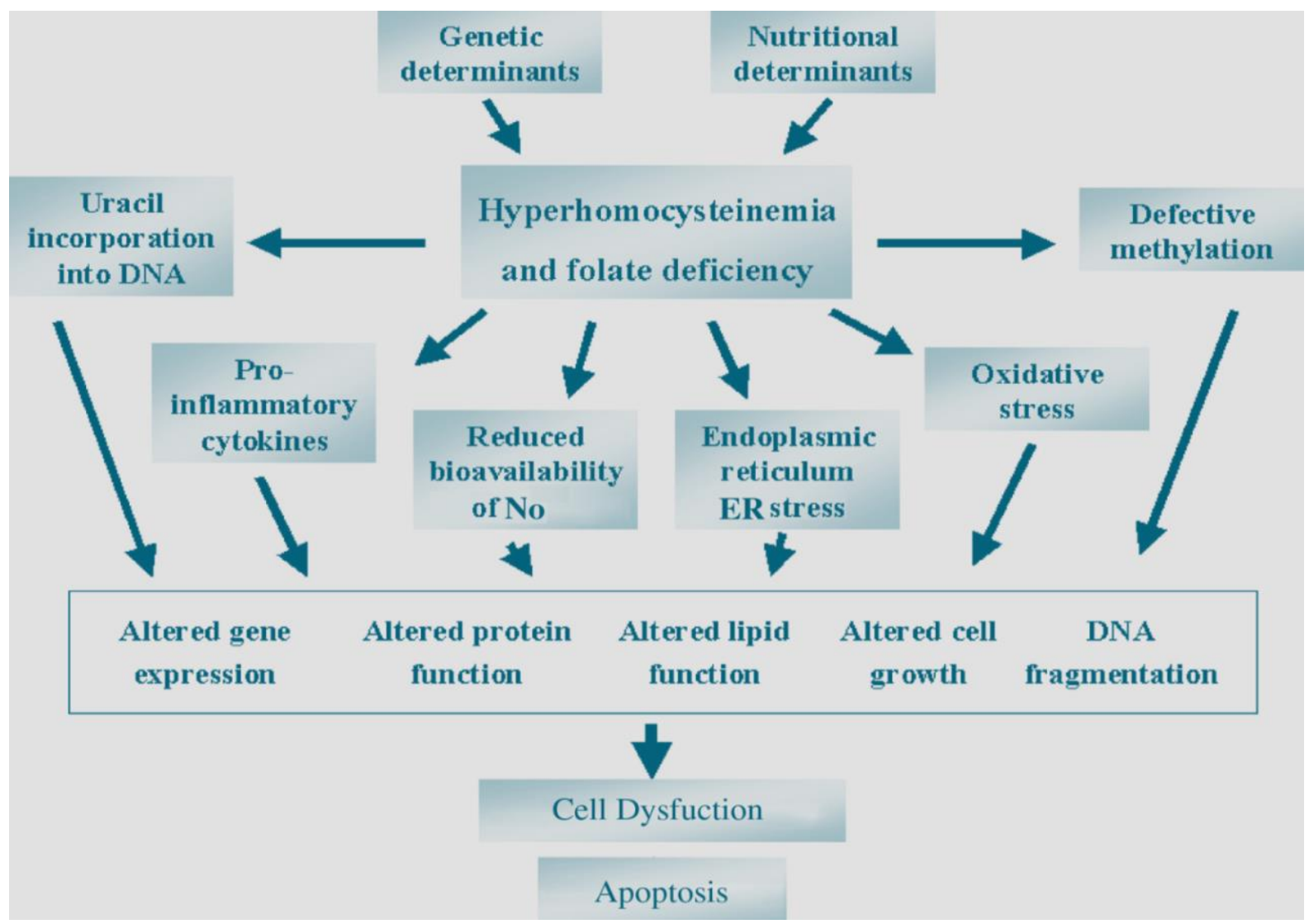

Figure 2: Cellular and molecular mechanisms of hyperhomocysteinemia-induced cell dysfunction.

This effect is mediated by an accelerated oxidative inactivation of NO and/or eNOS or by an increase in serum assymetric dimethylarginine, an endogenous inhibitor of eNOS. ${ }^{14}$ Third, hyperhomocysteinemia is found to be associated with the production of reactive oxygen species (ROS) in endothelial and smooth muscle cells. The mechanism of this oxidative stress relies either on auto-oxidation of the highly reactive thiol group of homocysteine or on the formation of intracellular superoxide and peroxyl radicals with concomitant inhibition of cellular antioxidant enzymes, such as superoxide dismutase and glutathione peroxidase. ${ }^{15,16}$ Fourth, a more recent concept concerns activation of the unfolded protein response (UPR) that is triggered when unfolded or misfolded proteins accumulate in the endoplasmic reticulum (ER). ${ }^{17}$ This ER stress induced after exposure to homocysteine ,further induces the expression of several molecular chaperones and other stress response proteins, which are aimed at restoring correct protein folding or retranslocating defective proteins back to the cytosol for degradation in the proteasomes. In case of a prolonged ER stress, the UPR activates apoptosis by various signaling pathways. ${ }^{18}$ Homocysteine-induced endothelial apoptosis probably also involves other mechanisms such as the classical p53 pathway. ${ }^{19}$ Furthermore, folate deficiency and genetically determined low MTHFR activity lead to an insufficient remethylation of homocysteine to methionine and a decreased SAM(S-adenosyl methionine) production and
SAM/SAH (S-adenosyl homocysteine) ratio. Insufficient availability of SAM then results in impaired methylation reactions, with multiple consequences. Defective methylation may lead to aberrant gene expression resulting in abnormal fetal development and malignant diseases. ${ }^{20}$ Finally, dietary folate deficiency and the resulting decreased cellular synthesis of 5,10methyleneTHF, as well as reduced MTHFR activity lead to an accumulation of dUMP (deoxy-uridyl monophosphate) and thus to an excessive incorporation of uracil into DNA, with the subsequent repair mechanisms increasing the risk of chromosomal breakage. $^{21,22}$

Many of these mechanisms are involved to cause hyperhomocysteinemia induced infertility. The aim of our study was to assess homocysteine levels in females of unexplained infertility and to study the effect of homocysteine level lowering agents on female infertility.

\section{METHODS}

The present study was carried out in unexplained infertile couples attending the outpatient department of Upper India Sugar Exchange Maternity Hospital, Department of Obstetrics \& Gynecology, attached to GSVM Medical college, Kanpur between October 2010 and September 2012 . 


\section{Inclusion criteria:}

1) Age 20-40 years old.

2) Cases of Unexplained infertility as diagnosed by ASRM guidelines $(2006)^{23}$ for standard infertility evaluation which includes:

a) Absence of male factor: Normal semen analysis as assessed by WHO 2010 guidelines

b) Adequate ovulation using either a mid luteal serum progesterone greater than $10 \mathrm{ng} / \mathrm{mL}$, urine testing documenting the LH surge or serial transvaginal ultrasounds to monitor the development and rupture of a dominant ovarian follicle.

c) Normal uterine cavity and patent tubes proved by hysterosalpingography or laparoscopy.

3) Fertile controls included women with one or more successful pregnancies but without gestational complications (e.g. intrauterine growth restriction, preeclampsia, stillbirth and abruptio placentae) and without any abortions.

\section{Exclusion criteria:}

Women with associated male factor of infertility, gross pelvic pathology, uncontrolled chronic disease e.g. diabetes mellitus, tuberculosis, history of chronic pelvic inflammatory disease, previous arterial and/or venous thrombosis or history of first degree relatives with arterial and/or venous thrombosis before the age of 65 years were excluded.

\section{Interventions:}

After taking consent \& informing the patients about the procedure, all patients were subjected to complete history taking, general examination, abdominal and pelvic examination, ultrasound examination for pelvic pathology, hysterosalpingography to rule out defects of the anatomical patency of the genital tract, venous blood sampling for serum follicular stimulating hormone, luteinizing hormone, TSH, progesterone \& prolactin, semen analysis of the patient's husband, venous blood sampling from patient's antecubital vein after overnight fasting centrifuged for $10 \mathrm{~min}$ at $2500 \mathrm{rpm}$ and plasma was stored till testing for serum homocysteine levels was done.

In our study we administered folic acid $5 \mathrm{mg}$, vitamin B12 500 microgram, vitamin B6 $5 \mathrm{mg}$ to patients with hyperhomocysteinemia (taking $13.5 \mu \mathrm{mol} / \mathrm{l}$ as cut off) and then re-assessed them for homocysteine levels after 6 weeks. The outcomes in terms of conceptions over a period of one year were also noted. The results were analyzed using data software like SPSS and MedCalc.

\section{RESULTS}

We compared mean serum homocysteine values among cases and controls and found that mean serum homocysteine levels were $17.27 \pm 9.49 \mu \mathrm{mol} / \mathrm{L}$ in the cases and $10.87 \mu \mathrm{moll} / \mathrm{L} \pm 4.27 \mu \mathrm{mol} / \mathrm{L}$ in the control group. Unpaired $\mathrm{t}$ - test was applied. ' $\mathrm{t}$ ' value was 3.37 and degree of freedom was 58 and the result was statistically significant $(\mathrm{p}$ value $=0.001$ i.e. $<0.05)$. Mean difference in values observed was 6.4 with $95 \%$ confidence interval of the mean difference between 2.60 and 10.20 .

Table 1: Comparison of serum homocysteine values among patients of unexplained infertility and fertile patients.

\begin{tabular}{|c|c|c|c|c|c|}
\hline $\begin{array}{l}\text { Serum } \\
\text { homocysteine } \\
(\mu \mathrm{mol} / \mathrm{L})\end{array}$ & $\begin{array}{l}\text { No. of } \\
\text { patients }\end{array}$ & Mean & $\begin{array}{l}\text { Std } \\
\text { dev. }\end{array}$ & $\mathbf{t}$ & $p$ \\
\hline Cases & 30 & 17.27 & $\begin{array}{l} \pm \\
9.49\end{array}$ & \multirow{2}{*}{3.37} & \multirow{2}{*}{0.001} \\
\hline Controls & 30 & 10.87 & $\begin{array}{l} \pm \\
4.27\end{array}$ & & \\
\hline
\end{tabular}

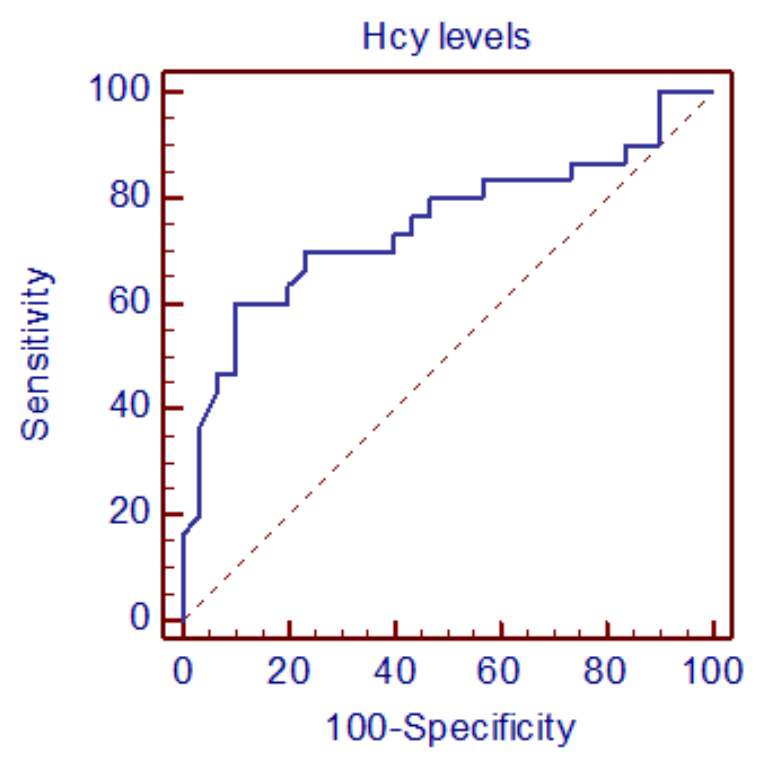

Figure 3: ROC curve analysis for serum homocysteine levels and infertility.

ROC curve (Figure 3) was applied to serum homocysteine values in the sample and area under the ROC curve was 0.749 which was significant. $\mathrm{z}$ value was 3.774 and significance of $p$ value 0.0002 was observed. Further sensitivity and specificity of the test was calculated at various values and a cut off value of 13.5 was calculated at which maximum sensitivity and specificity was observed (sensitivity $70 \%$ and specificity $76.7 \%$ ).

Majority $22(73.3 \%)$ of patients among the cases had hyperhomocysteinemia while majority 26(86.6\%) of controls had normal homocysteine values. 
Table 2: Distribution of patients according to homocysteine levels.

\begin{tabular}{|llll|l|}
\hline Homocysteine levels & No. of cases & $\%$ & No. of controls & $\%$ \\
\hline Patients with hyperhomocysteinemia & 22 & 73.33 & 4 & 13.33 \\
\hline $\begin{array}{l}\text { Patients with normal homocysteine } \\
\text { levels }\end{array}$ & 8 & 26.66 & 26 & 86.66 \\
\hline Total & 30 & 100 & 30 & 100 \\
\hline
\end{tabular}

These twenty two patients $(73.3 \%)$ among 30 cases, who were found to have serum homocysteine above the reference range value i.e. $>=13.5 \mu \mathrm{mol} / 1$, were given intervention in the form of 6 weeks therapy of homocysteine lowering agents. Paired ' $t$ ' test was applied to the samples taken before and after and tested for homocysteine levels.

Table 3: Serum homocysteine values before and after treatment intervention.

\begin{tabular}{|c|c|c|c|c|}
\hline & Mean $(n=22)$ & Standard Deviation & Paired ' $t$ ' & $p$ value \\
\hline Before treatment & 20.64 & \pm 8.77 & \multirow{2}{*}{7.24} & \multirow{2}{*}{0.0001} \\
\hline After treatment & 10.10 & \pm 4.12 & & \\
\hline
\end{tabular}

Mean serum homocysteine levels among the 22 cases were $20.64 \mu \mathrm{mol} / \mathrm{L} \pm 8.77 \mu \mathrm{mol} / \mathrm{L}$ before and 10.10 $\mu \mathrm{mol} / \mathrm{L} \pm 4.12 \mathrm{~mol} / \mathrm{L}$ after the treatment. Mean serum homocysteine lowering was $10.54 \mu \mathrm{mol} / \mathrm{L} \pm 6.83 \mu \mathrm{mol} / \mathrm{L}$. 95\% Confidence Intervals are between $7.51-13.5$ $\mu \mathrm{mol} / \mathrm{L}$. The result was highly significant $(p$ value < $0.001)$. This result showed that our intervention leads to significant reduction in serum homocysteine values.

Six of the 22 patients conceived in the follow up period of one year after the intervention. However, the patients are still under follow up as the pregnancy outcome of the patients is under study.

Table 4: Number of conceptions among the patients treated for hyperhomocysteinemia.

\begin{tabular}{|lll|}
\hline $\begin{array}{l}\text { No of patients with } \\
\text { hyperhomocysteinemia } \\
\text { treated }\end{array}$ & $\begin{array}{l}\text { Patients } \\
\text { conceived }\end{array}$ & $\%$ \\
\hline 22 & 6 & $27.27 \%$ \\
\hline
\end{tabular}

\section{DISCUSSION}

Homocysteine values have been found to be elevated significantly in patients with unexplained infertility by various statistical analyses in our study i.e. the independent ' $t$ ' test and ROC curve analysis. Findings of our study were also supported by the preliminary work by D'Uva et al on female reproduction. Their study revealed raised mean homocysteine levels of $21.05 \pm 8.78$ micromoles /litre in 20 women with unexplained sterility, $19.2 \pm 6.14 \mu \mathrm{mol} / 1$ for patients with recurrent pregnancy loss versus $7.85 \pm 3.31 \mu \mathrm{mol} / 1$ for controls. Their study gives an indication that infertility and recurrent pregnancy loss are a part of the continuum of the hyperhomocysteinemia induced adverse effects on female reproductive system. ${ }^{24}$
In studies by Bibi et al done in Pakistani women ,mean fasting homocysteine levels observed were significantly higher $(\mathrm{p}=0.04)$ in cases $(12.82 \pm 5.18$ micromoles /litre $)$ compared with controls $(9.735 \pm 1.80$ micromoles/litre) which was in concordance with our study. ${ }^{25}$

In our study among the infertile Indian females of unexplained infertility, we found the prevalence of hyperhomocysteinemia to be $73.3 \%$.Refsum $\mathrm{H}$ et al in a study of 100 people from Pune, India reported that $77 \%$ had plasma homocysteine concentrations $>15$ micromoles/litre. ${ }^{26}$

In our study intervention in the form of folic acid, vitamin B6, vitamin B12, and zinc lead to significant lowering of serum homocysteine levels. Shidfar F et al in their study concluded that folate supplementation decreases the serum levels of homocysteine. They studied the effect of folate supplementation versus placebo on 40 hyper-cholestrolemic subjects. Their results were similar to that of our study. ${ }^{27}$ In studies by Chait A et al supplementation of vitamin B6, B12 \& folate rich diet lead to significant reduction in homocysteine levels of studied group. $^{28}$

\section{CONCLUSIONS}

Serum homocysteine values were found to be significantly higher in the infertile females (17.29 \pm 9.49 micromoles/litre) as compared to their fertile controls (10.87 \pm 4.27 micromoles/litre). A critical level of 13.5 $\mu \mathrm{mol} / \mathrm{l}$ is suggested as the cut off for intervention by this study.

Higher than usual prevalence of homocystinemia in this cohort suggests the increased prevalence of micronutrient deficiency due to inadequate diet or dietary fads in the population. Micronutrient supplementation in the form of Vitamin B6, B12 and folic acid is helpful in reducing homocysteine values in patients with 
hyperhomocysteinemia. However, large scale studies are required to establish the role of hyperhomocysteinemia in unexplained infertility.

Funding: No funding sources

Competing interests: None declared

Ethical approval: Not required

\section{REFERENCES}

1. Gnoth C, Godehart E,Frank-Hermann $\mathrm{P}$, et al.Definition and prevalence of subfertility and infertility. Hum Reprod 2005;20:1144-7.

2. Barbieri RL. Induction of ovulation in infertile women with hyperandrogenism and insulin resistance, Am J Obstet Gynecol 2000;193:256-62.

3. Donderwinkel PF, Van der Vaart, Wolters VM. Treatment of patient with longstanding unexplained infertility with in vitro fertilization. Fertil Steril 2000;73:334-7.

4. Aboulghar M, Mansour R, Serour G: controlled ovarian hyperstimulation and intrauterine insemination for treatment of unexplained infertility should be limited to a maximum of three trials. Fertil Steril 2001;75:88-91.

5. Mira Aubuchon, Richard O. Burney, Danny J. Schust, Mylene W.M. Yao. Infertility and assisted reproductive technology, Berek and Novak's Gynecology.15th ed. South Asian edition, SAE : Wolters and Kluwer Health;2012:1139.

6. Martinelli I. Risk factors in venous thromboembolism. Thromb Haemostat 2001,86:395403.

7. Fatini C, Gensini F, Battagilini B, et al. Angiotensin converting enzyme DD genotype,angiotensin type 1 receptor $\mathrm{CC}$ genotype,and hyperhomocysteinemia increase first trimester fetal loss susceptibility. Blood Coagul Fibrinolysis 2000,11:657-62.

8. Wouters MG, Boers GH, Blom HJ. Hyperhomocysteinemia :a risk factor in women with unexplained recurrent pregnancy loss. Fertil Steril 1993,60:820-5.

9. Steegers Theunissen RP, Boers GH, et al. Maternal hyperhomocysteinemia a risk factor for neural tube defects? Metabolism 1994,43:1475-80.

10. Bertsch T, Mielke O, Holy S, Zimmer W, Casain W et al. Homocysteinemia in cerebrovascular disease: an independent risk factor for subcortical vascular encephalopathy. Clin Chem Lab Med 2001,39:721-4.

11. Hermann W, Knapp JP. Hyperhomocysteinemia: a new risk factor for degenerative diseases. Clin Lab 2002,48:471-81.

12. Forges T, Monnier - Barbarino P, Alberto JM, Guent - Rodriquez RM, Dava JL, Guent JL. Impact of folate and homocysteine metabolism on human reproduction health. Hum Reprod Update 2007; 13:225-38.

13. Poddar R, Sivasubramanian N, DiBello PM, et al. Homocysteine induces expression and secretion of monocyte chemoattractant protein-1 and interleukin-8 in human aortic endothelial cells: implications for vascular disease. Circulation 2001;103:2717-23.

14. Stuhlinger MC, Oka RK, Graf EE, et al. Endothelial dysfunction induced by hyperhomocysteinemia: Role of asymmetric dimethylarginine. Circulation 2003;108:933-8.

15. Starkebaum G, Harlan JM. Endothelial cell injury due to copper-catalyzed hydrogen peroxide generation from homocysteine. J Clin Invest 1986;77:1370-6.

16. Weiss N. Mechanisms of increased vascular oxidant stress in hyperhomocysteinemia and its impact on endothelial function. Curr Drug Metab 2005;6:27-36.

17. Kaufman RJ. Orchestrating the unfolded protein response in health and disease. J Clin Invest 2002;110:1389-98.

18. Xu C, Bailly-Maitre B, Reed JC. Endoplasmic reticulum stress: cell life and death decisions. J Clin Invest 2005;115:2656-64.

19. Lee NP, Cheng CY. Nitric oxide/nitric oxide synthase, spermatogenesis, and tight junction dynamics. Biol Reprod 2005;70:267-76.

20. Reik W, Walter J. Genomic imprinting: parental influence on the genome. Nat Rev Genet 2001;2:2132.

21. Blount BC, Mack MM, Wehr CM, et al. Folate deficiency causes uracil misincorporation into human DNA and chromosome breakage: implications for cancer and neuronal damage. Proc Natl Acad Sci USA 1997;94:3290-5.

22. Fenech M. The role of folic acid and Vitamin B12 in genomic stability of human cells. Mutat Res 2001;475:57-67.

23. The Practice Committee of the American Society for Reproductive Medicine, authors. Optimal evaluation of the infertile female. Fertil Steril 2006;86(5 suppl):S264-S267.

24. D' Uva M, Micco PD, Strina I, Alviggi C, Iannuzzo M, Ranieri A, et al. Hyperhomocysteinemia in Women with explained sterility or recurrent early pregnancy loss from southern Italy: a Preliminary report. Thrombosis J 2007;5:10.

25. Bibi S, Pir MA, et al. Hyperhomocyteinemia in women suffering from unexplained subfertility. Iranian J Reprod Medicine 2010;8:76-9.

26. Refsum H, Yajnik CS, Gadkari $M$, et al. Hyperhomocysteinemia and elevated methylmalonic acid indicate a high prevalence of cobalamin deficiency in Asian Indians. Am J Clin Nutr 2001;74:233-41.

27. Shidfar F, et al. Effect of folate supplementation on serum homocysteine and total antioxidant capacity in hypercholesterolemic adults. Arch Med Res 2009;40:380-6.

28. Chait A, Malinow MR, Nevin DN, Morris CD, Eastgard RL, Kris-Etherton P, et al. Increased dietary micronutrients decrease serum homocysteine concentrations in patients at high risk of 
cardiovascular disease. Am J Clin Nutr 1999;70:8817.
DOI: $10.5455 / 2320-1770$. ijrcog20130611

Cite this article as: Dubey P, Gupta N, Dwivedi S, Swaroop N, Lal P, Thawani V.

Hyperhomocysteinemia: a risk factor in unexplained infertility. Int J Reprod Contracept Obstet Gynecol 2013;2:165-71. 